\section{A preliminary study on antioxidant activities of saffron petal extracts in lambs}

\author{
Arash Omidi,1 Seddighe Rahdari, ${ }^{2}$ \\ Mohammad Hassanpour Fard3 \\ 1Department of Animal Health \\ Management, School of Veterinary \\ Medicine, Shiraz University, Shiraz; \\ 2Department of Animal Sciences, \\ Agriculture Faculty, Birjand University, \\ Birjand; 3Department of Pharmacology, \\ School of Medicine, Birjand University of \\ Medical Sciences, Iran
}

\section{Abstract}

This study assessed the effects of hydroalcoholic extract of saffron (Crocus sativus $\mathrm{L}$ ) petals on male lambs and was aimed at evaluating the antioxidant activity of this extract during a 15day period. Fourteen male lambs were divided randomly into three treatment groups $(n=4$ each) and a control group (normal saline; $\mathrm{n}=2$ ). Saffron petal extract at 500, 1000 and 1500 $\mathrm{mg} / \mathrm{kg}$ was administered by gavage once daily on days 1 and 3 of the experiment for treatment groups. Blood samples were obtained on days 6 and 14 of the study. Serum and plasma were stored at $-21^{\circ} \mathrm{C}$ for further analysis. Heart rate, respiratory rate, rectal temperatures and body weight of each lamb were recorded in the distinctive intervals from day 0 to the last day of the study. Total antioxidant capacity levels were increased significantly with any dose of the extract on the first day of sampling. There was no statistical difference in the levels of malondialdehyde and total thiol between the treatment and the control group son the $6^{\text {th }}$ and the $14^{\text {th }}$ days of the experiment. Also, no significant differences were identified in the concentrations of glucose, blood urea nitrogen, creatinine, uric acid, total cholesterol, triglyceride, albumin, globulin, total protein, aspartate aminotransferase, alanine aminotransferase, alkaline phosphatase, gamma glutamyle transferase, total bilirubin, direct and indirect bilirubin between control and treatment groups in male lambs during this study. Recorded vital signs and body weight in control and treatment groups showed no significant differences. The present results suggest that the hydroalcoholic extract of saffron petals has antioxidant activity in sheep.

\section{Introduction}

Crocus sativus L., commonly known as saf- fron, is a bulbous perennial stem less plant of the Iridaceae family. Saffron, widely cultivated in Iran, is well adapted to arid and semi-arid lands and produces stigmas annually. It is also adaptable to temperate and sub-tropical climates. Saffron has been used in folk medicine for more than 3000 years. In common, the harvested stigma is used as a food spice or as an herb with medicinal properties. Saffron petal is a by-product of saffron and is usually discarded as a waste. ${ }^{1}$ In some areas the remaining by-products of saffron or saffron petals is fed to flocks of sheep and goats. A number of studies indicate that ethanol extract of saffron petals possesses antidepressant, ${ }^{2-5}$ anti-inflammatory, antinociceptive, ${ }^{6-8}$ antihypertensive), ${ }^{9}$ anti-cancer and antitumor activities.10,11 Phenolic compounds, among which are flavonoids and anthocyanins, ${ }^{12}$ are likely the biologically active components of the saffron petal and have been associated with the health benefits in human and animals.13-15 The antioxidant and antimicrobial properties of saffron have been noticed in recent years. 16 The beneficial effects derived from phenolic compounds have been attributed to their antioxidant activity. 17 The present study was undertaken firstly to investigate the oxidant/ antioxidant status in male lambs receiving the petal extract and secondly to evaluate whether the hydroalcoholic extract of saffron petal has any adverse effect on the health of the male lambs.

To our knowledge this is the first study of effects of saffron petals on serum biochemical parameters of sheep.

\section{Materials and Methods}

\section{Animals}

The study was conducted on fourteen 4-6 month-old Baluchi male lambs, weighing $38.40 \pm 1.04 \mathrm{~kg}$, during November and December, 2011. In this study, 12 lambs were divided randomly into 3 treatment groups $(\mathrm{n}=4$ each).vTwo lambs were considered to be the control group; they received normal saline as placebo. The treatment groups I, II and III, respectively, received 500, 1000 and 1500 $\mathrm{mg} / \mathrm{kg}$ saffron petal extract by gavage once daily at days 1 and 3 of the experiment. The animals were treated against internal and external parasites with Albendazole (Damloran Company, Borujerd, Iran) 10 $\mathrm{mg} / \mathrm{kg}$, and Ivermectin (Razak company, Tehran, Iran) $0.22 \mathrm{mg} / \mathrm{kg}, 30$ days prior the beginning of the study.

\section{Preparing the petal extract}

Crocus sativus L. petals were collected from Torbat-Heidariye district $\left(35.27^{\circ}\right.$ north latitude, $59.22^{\circ}$ east longitude, about 1330 meters above sea level) in Khorasan-Razavi province,
Correspondence: Arash 0midi, Department of Animal Health Management, School of Veterinary Medicine, Shiraz University, Shiraz 71345-1731, Iran.

Tel. +98.711.613.8745 - Fax: +98.711.228.6940

E-mail: aomidi@shirazu.ac.ir

Key words: Saffron petal extract, antioxidant activity, lamb, biochemical variable.

Acknowledgements: this article was extracted from the thesis prepared by Seddighe Rahdari to fulfill the requirements required for earning the Master of Science degree in animal physiology. The manuscript was edited by Manuscript Editor Online.

Funding: the authors gratefully acknowledge the financial support for this work that was provided by the Vice Chancellor for Research and Saffron Research Group at Birjand University.

Received for publication: 6 November 2013. Revision received: 7 December 2013.

Accepted for publication: 19 December 2013

This work is licensed under a Creative Commons Attribution NonCommercial 3.0 License (CC BYNC 3.0).

(C) Copyright A. Omidi et al., 2014

Licensee PAGEPress srl, Italy

Veterinary Science Development 2014; 4:5161

doi:10.4081/vsd.2014.5161

North-east of Iran, during autumn 2011 (Figure 1). The samples were identified by the Agricultural Faculty of Birjand University, Iran. The voucher number specimen (No. 74/1525) was deposited in the herbarium of Birjand University, Iran. To prepare the petal extracts, the samples were dried in shadow and then pulverized with a grinder (Hamilton Beach Brand, Canada). Hydroalcoholic extract was prepared by using $50 \mathrm{~g}$ of the dried powder in $1000 \mathrm{~mL}$ of $80 \% \mathrm{v} / \mathrm{v}$ ethanol and shaking for 24 h. Then, the mixture was filtered through No. 1 Whatman filter paper and was oven dried at $40^{\circ} \mathrm{C}$ for 24 hours. The final powdered extract was then weighed to calculate the yield. The yield $(w / w)$ of the ethanolic petal extract was $30 \%$.

\section{Clinical study}

The safety of the extract was assessed in the animals by monitoring the vital signs and clinical findings as well as performing laboratory tests. The lambs were closely observed twice a day for any sign of illness. Heart and respiratory rates, rectal temperatures and live weights of the lambs were recorded for 15-days (10:00 AM) from day 0 of the study onwards. Blood samples were obtained by jugular venepuncture into plain and EDTA tubes at days 6 and 14 
of the experiment between 8:00 and 9:00 am. The experiment was approved by the animal welfare committee of the Agricultural Faculty of Birjand University, Iran.

\section{Laboratory analysis}

Serum and plasma were separated by centrifugation of the samples at $3000 \times g$ for $15 \mathrm{~min}$ at room temperature, and were stored at $-21^{\circ} \mathrm{C}$ for further analysis. Serum oxidant status was evaluated by measuring malondialdehyde (MDA) and serum antioxidant status was assessed by measuring total thiol (T-SH) levels and total antioxidant capacity (TAC). MDA was determined according to the method described by Ohkawa et al. ${ }^{18}$ MDA is formed as an end product of lipid peroxidation, which reacts with the TBA (thiobarbituric acid) reagent under

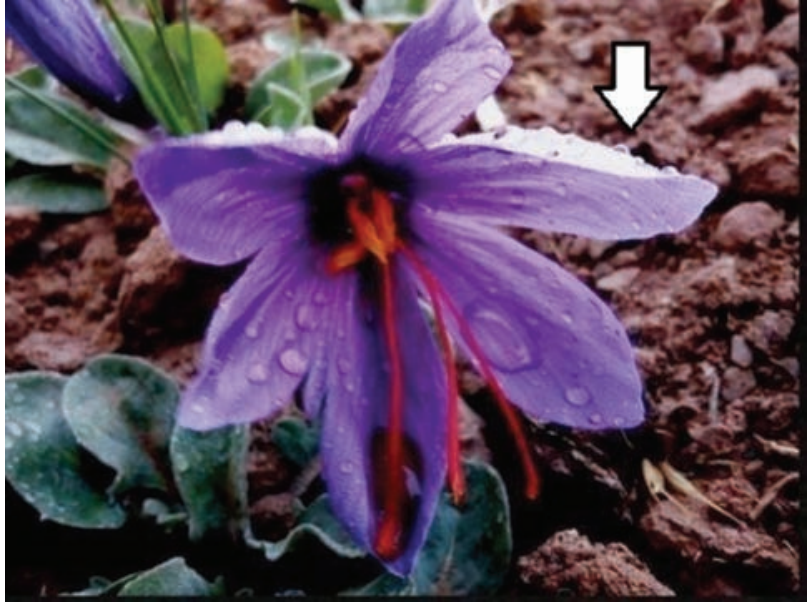

Figure 1. The white arrow indicates saffron petal, which is used in the experiment.

Table 1. The concentration of some blood biochemical variables in control and treatment groups in healthy male lambs (mean \pm S.E.M).

\begin{tabular}{|c|c|c|c|c|c|c|c|}
\hline Variable & Unit & D & Control & Treatment I & Treatment II & Treatment III & Sig. \\
\hline MDA & $\mu \mathrm{mol} / \mathrm{L}$ & $\begin{array}{c}6 \\
14\end{array}$ & $\begin{array}{l}2.29 \pm 0.27 \\
2.92 \pm 0.35\end{array}$ & $\begin{array}{l}2.67 \pm 0.23 \\
2.02 \pm 0.29\end{array}$ & $\begin{array}{l}2.53 \pm 0.21 \\
2.15 \pm 0.11\end{array}$ & $\begin{array}{l}2.61 \pm 0.17 \\
2.46 \pm 0.16\end{array}$ & $\begin{array}{l}\text { N.S } \\
\text { N.S }\end{array}$ \\
\hline T-SH & $\mu \mathrm{mol} / \mathrm{L}$ & $\begin{array}{c}6 \\
14\end{array}$ & $\begin{array}{c}19.99 \pm 2.79 \\
34.63 \pm 5.96\end{array}$ & $\begin{array}{l}24.25 \pm 1.12 \\
32.44 \pm 3.24\end{array}$ & $\begin{array}{l}25.46 \pm 1.51 \\
43.24 \pm 5.58\end{array}$ & $\begin{array}{l}21.69 \pm 3.26 \\
34.10 \pm 4.48\end{array}$ & $\begin{array}{l}\text { N.S } \\
\text { N.S }\end{array}$ \\
\hline $\mathrm{TAC}$ & $\mu \mathrm{mol} / \mathrm{L}$ & $\begin{array}{c}6 \\
14\end{array}$ & $\begin{array}{l}321.07 \pm 6.14 \mathrm{a} \\
322.83 \pm 78.15\end{array}$ & $\begin{array}{l}400.55 \pm 22.76 \mathrm{~b} \\
345.22 \pm 26.71\end{array}$ & $\begin{array}{c}376.84 \pm 15.78 b \\
427.33 \pm 20.02\end{array}$ & $\begin{array}{c}419.87 \pm 18.41 \mathrm{~b} \\
427.33 \pm 37.77\end{array}$ & $\begin{array}{c}* \\
\text { N.S }\end{array}$ \\
\hline Glucose & mg/dL & $\begin{array}{c}6 \\
14\end{array}$ & $\begin{array}{c}87.00 \pm 8.00 \\
77.500 \pm 1.500\end{array}$ & $\begin{array}{c}92.00 \pm 13.78 \\
84.00 \pm 6.94\end{array}$ & $\begin{array}{l}81.50 \pm 4.21 \\
74.25 \pm 2.71\end{array}$ & $\begin{array}{l}83.50 \pm 2.60 \\
71.00 \pm 3.31\end{array}$ & $\begin{array}{l}\text { NS } \\
\text { NS }\end{array}$ \\
\hline BUN & $\mathrm{mg} / \mathrm{dL}$ & $\begin{array}{c}6 \\
14\end{array}$ & $\begin{array}{l}7.50 \pm 3.33 \\
6.04 \pm 1.87\end{array}$ & $\begin{array}{l}11.11 \pm 1.60 \\
10.97 \pm 0.77\end{array}$ & $\begin{array}{c}11.66 \pm 1.67 \\
6.97 \pm 1.10\end{array}$ & $\begin{array}{c}10.00 \pm 5.06 \\
9.06 \pm 0.74\end{array}$ & $\begin{array}{l}\text { NS } \\
\text { NS }\end{array}$ \\
\hline Urea & mg/dL & $\begin{array}{c}6 \\
14\end{array}$ & $\begin{array}{c}18.00 \pm 8.00 \\
14.500 \pm 4.500\end{array}$ & $\begin{array}{l}23.75 \pm 3.99 \\
24.00 \pm 2.68\end{array}$ & $\begin{array}{l}28.00 \pm 4.02 \\
16.75 \pm 2.65\end{array}$ & $\begin{array}{l}24.00 \pm 3.72 \\
21.75 \pm 1.80\end{array}$ & $\begin{array}{l}\text { NS } \\
\text { NS }\end{array}$ \\
\hline Creatinin & $\mathrm{mg} / \mathrm{dL}$ & $\begin{array}{c}6 \\
14\end{array}$ & $\begin{array}{l}1.10 \pm 0.20 \\
1.05 \pm 0.25\end{array}$ & $\begin{array}{l}0.93 \pm 0.21 \\
0.82 \pm 0.13\end{array}$ & $\begin{array}{l}0.85 \pm 0.13 \\
0.90 \pm 0.20\end{array}$ & $\begin{array}{c}0.90 \pm 0.11 \\
0.82 \pm 0.04\end{array}$ & $\begin{array}{l}\text { NS } \\
\text { NS }\end{array}$ \\
\hline Uric acid & $\mathrm{mg} / \mathrm{dL}$ & $\begin{array}{c}6 \\
14\end{array}$ & $\begin{array}{l}1.05 \pm 0.15 \\
1.70 \pm 0.60\end{array}$ & $\begin{array}{l}1.07 \pm 0.44 \\
0.70 \pm 0.07\end{array}$ & $\begin{array}{l}1.07 \pm 0.28 \\
2.70 \pm 1.48\end{array}$ & $\begin{array}{l}0.68 \pm 0.19 \\
1.02 \pm 0.34\end{array}$ & $\begin{array}{l}\text { NS } \\
\text { NS }\end{array}$ \\
\hline Total cholesterol & $\mathrm{mg} / \mathrm{dL}$ & $\begin{array}{c}6 \\
14\end{array}$ & $\begin{array}{l}56.00 \pm 8.00 \\
30.50 \pm 5.50\end{array}$ & $\begin{array}{l}52.75 \pm 2.87 \\
35.75 \pm 1.65\end{array}$ & $\begin{array}{l}53.75 \pm 4.13 \\
38.00 \pm 4.14\end{array}$ & $\begin{array}{l}46.00 \pm 2.04 \\
36.75 \pm 6.94\end{array}$ & $\begin{array}{l}\text { NS } \\
\text { NS }\end{array}$ \\
\hline Triglyceride & $\mathrm{mg} / \mathrm{dL}$ & $\begin{array}{c}6 \\
14 \\
\end{array}$ & $\begin{array}{l}32.00 \pm 2.00 \\
19.00 \pm 1.00\end{array}$ & $\begin{array}{l}38.50 \pm 5.33 \\
33.25 \pm 5.28\end{array}$ & $\begin{array}{l}37.00 \pm 2.38 \\
44.00 \pm 14.54\end{array}$ & $\begin{array}{l}30.75 \pm 6.87 \\
30.00 \pm 2.94\end{array}$ & $\begin{array}{l}\text { NS } \\
\text { NS }\end{array}$ \\
\hline Albumin & $\mathrm{g} / \mathrm{dL}$ & $\begin{array}{c}6 \\
14\end{array}$ & $\begin{array}{l}3.80 \pm 0.10 \\
3.35 \pm 0.05\end{array}$ & $\begin{array}{l}3.73 \pm 0.05 \\
3.52 \pm 0.25\end{array}$ & $\begin{array}{l}4.00 \pm 0.09 \\
3.62 \pm 0.17\end{array}$ & $\begin{array}{l}3.65 \pm 0.05 \\
3.60 \pm 0.07\end{array}$ & $\begin{array}{l}\text { NS } \\
\text { NS }\end{array}$ \\
\hline Globulin & $\mathrm{g} / \mathrm{dL}$ & $\begin{array}{c}6 \\
14 \\
\end{array}$ & $\begin{array}{l}4.75 \pm 0.55 \\
4.80 \pm 0.20\end{array}$ & $\begin{array}{l}4.40 \pm 0.10 \\
4.10 \pm 0.12\end{array}$ & $\begin{array}{l}4.97 \pm 0.27 \\
4.50 \pm 0.24\end{array}$ & $\begin{array}{l}5.05 \pm 0.48 \\
4.22 \pm 0.23\end{array}$ & $\begin{array}{l}\text { NS } \\
\text { NS }\end{array}$ \\
\hline A:G ratio & $\%$ & $\begin{array}{c}6 \\
14\end{array}$ & $\begin{array}{l}0.81 \pm 0.11 \\
0.69 \pm 0.03\end{array}$ & $\begin{array}{l}0.84 \pm 0.02 \\
0.86 \pm 0.03\end{array}$ & $\begin{array}{l}0.81 \pm 0.04 \\
0.81 \pm 0.07\end{array}$ & $\begin{array}{l}0.74 \pm 0.06 \\
0.85 \pm 0.04\end{array}$ & $\begin{array}{l}\text { NS } \\
\text { NS }\end{array}$ \\
\hline Total protein & $\mathrm{g} / \mathrm{dL}$ & $\begin{array}{c}6 \\
14\end{array}$ & $\begin{array}{l}8.55 \pm 0.45 \\
8.15 \pm 0.15\end{array}$ & $\begin{array}{l}8.13 \pm 0.09 \\
7.62 \pm 0.10\end{array}$ & $\begin{array}{c}8.98 \pm 0.31 \\
8.12 \pm 0.0 .23\end{array}$ & $\begin{array}{l}8.70 \pm 0.48 \\
7.82 \pm 0.26\end{array}$ & $\begin{array}{l}\text { NS } \\
\text { NS }\end{array}$ \\
\hline AST1 & $\mathrm{IU} / \mathrm{L}$ & $\begin{array}{c}6 \\
14\end{array}$ & $\begin{array}{c}102.00 \pm 20.00 \\
90.00 \pm 6.00\end{array}$ & $\begin{array}{c}115.75 \pm 8.75 \\
107.75 \pm 15.17\end{array}$ & $\begin{array}{c}178.50 \pm 43.57 \\
127.75 \pm 4.50\end{array}$ & $\begin{array}{l}109.75 \pm 8.70 \\
95.00 \pm 17.71\end{array}$ & $\begin{array}{l}\text { NS } \\
\text { NS }\end{array}$ \\
\hline $\mathrm{ALT}^{2}$ & IU/L & $\begin{array}{c}6 \\
14\end{array}$ & $\begin{array}{c}12.50 \pm 0.50 \\
27.50 \pm-4.26\end{array}$ & $\begin{array}{l}23.75 \pm 4.80 \\
21.50 \pm 5.78\end{array}$ & $\begin{array}{c}45.00 \pm 14.29 \\
24.75 \pm 5.40\end{array}$ & $\begin{array}{l}31.25 \pm 3.57 \\
25.00 \pm 4.70\end{array}$ & $\begin{array}{l}\text { NS } \\
\text { NS }\end{array}$ \\
\hline ALP3 & $\mathrm{IU} / \mathrm{L}$ & $\begin{array}{c}6 \\
14\end{array}$ & $\begin{array}{l}899.00 \pm 408.00 \\
863.50 \pm 428.50\end{array}$ & $\begin{array}{l}724.00 \pm 41.67 \\
644.50 \pm 57.12\end{array}$ & $\begin{array}{l}727.00 \pm 36.92 \\
526.75 \pm 53.28\end{array}$ & $\begin{array}{l}723.75 \pm 132.65 \\
659.75 \pm 114.33\end{array}$ & $\begin{array}{l}\text { NS } \\
\text { NS }\end{array}$ \\
\hline $\mathrm{Y-GT4}$ & IU/L & $\begin{array}{c}6 \\
14\end{array}$ & $\begin{array}{c}57.90 \pm 2.60 \\
49.20 \pm 18.70\end{array}$ & $\begin{array}{l}85.30 \pm 12.36 \\
77.92 \pm 11.71\end{array}$ & $\begin{array}{l}72.43 \pm 5.12 \\
57.40 \pm 6.18\end{array}$ & $\begin{array}{l}74.50 \pm 7.08 \\
67.45 \pm 4.82\end{array}$ & $\begin{array}{l}\text { NS } \\
\text { NS }\end{array}$ \\
\hline Direct Bilirubin & $\mathrm{mg} / \mathrm{dL}$ & $\begin{array}{c}6 \\
14\end{array}$ & $\begin{array}{l}0.10 \pm 0.00 \\
0.10 \pm 0.00\end{array}$ & $\begin{array}{l}0.10 \pm 0.00 \\
0.10 \pm 0.00\end{array}$ & $\begin{array}{l}0.10 \pm 0.00 \\
0.10 \pm 0.00\end{array}$ & $\begin{array}{l}0.10 \pm 0.00 \\
0.10 \pm 0.00\end{array}$ & $\begin{array}{l}\text { NS } \\
\text { NS }\end{array}$ \\
\hline Indirect Bilirubin & mg/dL & $\begin{array}{c}6 \\
14\end{array}$ & $\begin{array}{l}0.25 \pm 0.05 \\
0.55 \pm 0.25\end{array}$ & $\begin{array}{l}0.20 \pm 0.04 \\
0.20 \pm 0.04\end{array}$ & $\begin{array}{l}0.12 \pm 0.02 \\
0.30 \pm 0.16\end{array}$ & $\begin{array}{l}0.12 \pm 0.02 \\
0.15 \pm 0.05\end{array}$ & $\begin{array}{l}\text { NS } \\
\text { NS }\end{array}$ \\
\hline Total Bilirubin & $\mathrm{mg} / \mathrm{dL}$ & $\begin{array}{c}6 \\
14\end{array}$ & $\begin{array}{l}0.35 \pm 0.05 \\
0.65 \pm 0.25\end{array}$ & $\begin{array}{l}0.30 \pm 0.04 \\
0.30 \pm 0.04\end{array}$ & $\begin{array}{c}0.22 \pm 0.02 \\
0.40 \pm 0.16\end{array}$ & $\begin{array}{c}0.22 \pm 0.02 \\
0.25 \pm 0.05\end{array}$ & $\begin{array}{l}\text { NS } \\
\text { NS }\end{array}$ \\
\hline
\end{tabular}

\footnotetext{
a,bDifferent superscripts letters indicate significant differences; ${ }^{1}$ Aspartate aminotransferase; ${ }^{2}$ Alanine aminotransferase; ${ }^{3 A l k a l i n e ~ p h o s p h a t a s e ; ~}{ }^{4} \gamma$-Glutamyltransferase. ${ }^{*} \mathrm{P} \leq 0.05$; NS, P $>0.05$. $\mathrm{D}=\mathrm{day}$.
} 
Table 2. Recorded vital signs and body weight during the experiment in control and treatment groups in healthy male lambs (mean \pm S.E.M).

\begin{tabular}{|c|c|c|c|c|c|c|}
\hline Variable & D & Control & Treatment I & Treatment II & Treatment III & Sig. \\
\hline $\begin{array}{l}\text { Heart rates } \\
\text { (Beats/minute) }\end{array}$ & $\begin{array}{c}2 \\
4 \\
7 \\
9 \\
11 \\
13 \\
15\end{array}$ & $\begin{array}{c}114.00 \pm 6.00 \\
120.00 \pm 0.00 \\
104.00 \pm 4.00 \\
116.00 \pm 4.00 \mathrm{a} \\
100.00 \pm 4.00 \\
150.00 \pm 22.00 \\
108.00 \pm 4.00\end{array}$ & $\begin{array}{c}120.00 \pm 5.88 \\
121.00 \pm 1.00 \\
96.00 \pm 3.65 \\
109.00 \pm 4.12 \mathrm{~b} \\
106.00 \pm 2.00 \\
132.00 \pm 8.48 \\
103.00 \pm 3.00\end{array}$ & $\begin{array}{c}94.00 \pm 6.21 \\
120.00 \pm 6.32 \\
98.00 \pm 7.39 \\
114.00 \pm 8.71 \mathrm{a} \\
97.00 \pm 3.78 \\
124.00 \pm 8.48 \\
95.00 \pm 2.51\end{array}$ & $\begin{array}{c}99.00 \pm 3.78 \\
129.00 \pm 8.73 \\
95.00 \pm 3.00 \\
108.00 \pm 6.92 \mathrm{~b} \\
103.00 \pm 3.00 \\
122.00 \pm 7.74 \\
99.00 \pm 4.43\end{array}$ & $\begin{array}{c}\text { NS } \\
\text { NS } \\
\text { NS } \\
* \\
\text { NS } \\
\text { NS } \\
\text { NS }\end{array}$ \\
\hline $\begin{array}{l}\text { Respiratory rates } \\
\text { (Beats/minute) }\end{array}$ & $\begin{array}{c}2 \\
4 \\
7 \\
9 \\
11 \\
13 \\
15\end{array}$ & $\begin{array}{c}36.00 \pm 4.00 \\
42.00 \pm 6.00 \\
38.00 \pm 6.00 \\
34.00 \pm 2.00 \\
38.00 \pm 2.00 \\
30.00 \pm 2.00 \\
30.00 \pm 10.00\end{array}$ & $\begin{array}{l}41.00 \pm 2.51 \\
41.00 \pm 2.51 \\
39.00 \pm 1.91 \\
39.00 \pm 3.00 \\
36.00 \pm 1.63 \\
30.00 \pm 3.46 \\
25.00 \pm 1.00\end{array}$ & $\begin{array}{l}42.00 \pm 3.83 \\
38.00 \pm 2.00 \\
35.00 \pm 1.91 \\
39.00 \pm 1.91 \\
38.00 \pm 2.00 \\
28.00 \pm 2.82 \\
26.00 \pm 1.15\end{array}$ & $\begin{array}{l}35.00 \pm 2.51 \\
38.00 \pm 2.58 \\
35.00 \pm 3.41 \\
33.00 \pm 3.00 \\
38.00 \pm 2.58 \\
32.00 \pm 3.65 \\
28.00 \pm 5.41\end{array}$ & $\begin{array}{l}\text { NS } \\
\text { NS } \\
\text { NS } \\
\text { NS } \\
\text { NS } \\
\text { NS } \\
\text { NS }\end{array}$ \\
\hline $\begin{array}{l}\text { Rectal temperatures } \\
\left(\mathrm{C}^{\circ}\right)\end{array}$ & $\begin{array}{c}2 \\
4 \\
7 \\
9 \\
11 \\
13 \\
15\end{array}$ & $\begin{array}{l}39.70 \pm 0.50 \\
39.85 \pm 0.35 \\
39.80 \pm 0.40 \\
40.05 \pm 0.25 \\
39.50 \pm 0.10 \\
39.75 \pm 0.05 \\
39.65 \pm 0.15\end{array}$ & $\begin{array}{l}40.02 \pm 0.10 \\
39.67 \pm 0.11 \\
39.90 \pm 0.20 \\
40.07 \pm 0.27 \\
39.35 \pm 0.08 \\
39.72 \pm 0.02 \\
39.67 \pm 0.06\end{array}$ & $\begin{array}{l}39.47 \pm 0.22 \\
39.40 \pm 0.17 \\
39.40 \pm 0.14 \\
39.80 \pm 0.22 \\
39.32 \pm 0.30 \\
39.60 \pm 0.10 \\
39.60 \pm 0.10\end{array}$ & $\begin{array}{l}39.52 \pm 0.33 \\
39.50 \pm 0.12 \\
39.45 \pm 0.27 \\
39.62 \pm 0.13 \\
39.40 \pm 0.18 \\
39.25 \pm 0.27 \\
39.70 \pm 0.21\end{array}$ & $\begin{array}{l}\text { NS } \\
\text { NS } \\
\text { NS } \\
\text { NS } \\
\text { NS } \\
\text { NS } \\
\text { NS }\end{array}$ \\
\hline Body weight (kg) & $\begin{array}{c}0 \\
7 \\
13\end{array}$ & $\begin{array}{l}38.60 \pm 1.90 \\
37.20 \pm 1.80 \\
39.40 \pm 2.10\end{array}$ & $\begin{array}{l}38.87 \pm 2.35 \\
36.50 \pm 2.21 \\
38.52 \pm 2.16\end{array}$ & $\begin{array}{l}39.37 \pm 2.50 \\
36.72 \pm 2.33 \\
38.82 \pm 2.31\end{array}$ & $\begin{array}{l}36.85 \pm 1.71 \\
34.50 \pm 1.55 \\
37.22 \pm 1.47\end{array}$ & $\begin{array}{l}\text { NS } \\
\text { NS } \\
\text { NS }\end{array}$ \\
\hline
\end{tabular}

a,bDifferent superscripts letters indicate significant differences. $*$ P $\leq 0.05 ; \mathrm{NS}, \mathrm{P}>0.05$. D, day.

acidic conditions to generate a pink-colored product. Total thiol level was measured according to the method of Sedlak and Lindsay. ${ }^{19}$ Antioxidant status was evaluated using ferric reducing antioxidant power (FRAP) assay. ${ }^{20}$ The concentrations of glucose, blood urea nitrogen (BUN), creatinine, uric acid, total cholesterol, triglyceride, albumin, total protein, total and direct bilirubin as well as the activities of aspartateaminotransferase (AST), alanine aminotransferase (ALT), alkaline phosphatase (ALP), gamma glutamyle transferase (GGT) were measured by an autoanalyser apparatus (Prestige 24i, Japan).21 Serum globulin was calculated by subtracting the serum albumin from serum total protein. Serum albumin: globulin ratio (A:G ratio) was calculated by dividing the values of serum albumin by serum globulin. Also serum indirect bilirubin was calculated by subtracting the serum direct bilirubin from serum total bilirubin.

\section{Statistical analysis}

All data were analyzed using SPSS 16/PC software. Statistical analysis was performed using one-way ANOVA followed by TukeyKramer multiple range test for multiple comparisons. Statistical significance was set at $\mathrm{P}<0.05 .22$

\section{Results}

The results are shown in Tables 1 and 2 . The plasma levels of TAC were significantly higher on the $6^{\text {th }}$ day of the experiment for lambs received saffron petal $(\mathrm{P}=0.01)$ compared to those of the control lambs (Table 1). There were no differences in the MDA levels between the control and treatment groups at days 6 and 14 of the experiment $(\mathrm{P}>0.05)$. There was no difference in the T-SH levels between the control and treatment groups on the $6^{\text {th }}$ and the $14^{\text {th }}$ days $(\mathrm{P}>0.05)$. No significant differences were identified in the concentration of glucose, BUN, creatinine, uric acid, total cholesterol, triglyceride, albumin, globulin, total protein, AST, ALT, ALP, GGT, total bilirubin, direct and indirect bilirubin between the control and treatment groups in male lambs during this study (Table 1). The vital signs and body weights showed no significant differences during the experiment in control and treatment groups (Table 2).

\section{Discussion}

Processes or reactions involving in production of reactive oxygen and nitrogen species (ROS and RNS) can potentially make harmful effects on weight. Compounds with antioxidant capacity are capable of protecting biological systems against oxidative stress. In the present study, total antioxidant capacity levels were increased significantly with either dose of the saffron petal extract at the first day of sampling. In the present study there were no differences in the MDA levels between the treatment and the control groups. MDA is a three carbon, low molecular weight aldehyde that can be produced from free radical attack on polyunsaturated fatty acids of biological membranes. Therefore, it is a frequently measured biomarker of oxidative stress. ${ }^{23}$ The results of this investigation showed no difference in the total thiol levels. The major part of thiol in plasma is derived from proteins, especially albumin, and they are susceptible to oxidation. According to the results of this study, significantly higher serum TAC concentration in the treatment groups compared to that of control sheep reflects a higher total antioxidant capacity in lambs that received saffron petals. In this study there were not any condition of disease or disorders based on clinical and laboratory findings and the extract of saffron petal, up to $1500 \mathrm{mg} / \mathrm{kg}$ body weight, did not cause any adverse effect on health status of male lambs. It can be concluded that the saffron petal extract could have an antioxidant effect in healthy sheep. So it can be assumed that saffron petal extract can help sheep to combat oxidative stress in many pathological conditions. Saffron stigma is the world's most costly spices but saffron petal costs little. Saffron petals are the main by-product of saffron processing which is produced in large amounts annually in Iran and is usually discarded.1,24 The main overall finding from this study is that petal of Crocus sativus may be useful for its antioxidant benefit in lambs. The extracts of saffron petals may possess antioxidant activities in sheep. In some cases, the 
remaining saffron farms are eaten by sheep. Due to antioxidant effects, it is recommended that saffron petals in the remaining fields be used to feed livestock. However, further studies are needed to assess the antioxidant activity of saffron petals in various diseases and poisonings of sheep. Phenolic compounds are likely to be the biologically active components of the petals. ${ }^{24}$ Kaempferol, isolated from the fresh flower petals of saffron has also been mentioned as the important component.8,25 Kaempferol was effective in scavenging free radicals. ${ }^{26}$ Materials rich in phenols can retard oxidative degradation of lipids and improve the quality and nutritional value of food. Livestock feeds are subjected to oxidative spoilage. Further studies on improving the quality and nutritional value of the feed mixed with saffron petals is recommended.

\section{References}

1. Eskandari-Torbaghan MR, Kamar AA, Astarei A. Effect of saffron (crocus sativus L.) petals on germination and primary growth of cotton (gossypiumhirsutum L.). Acta Hort 2007;739:87-91.

2. Karimi G, Hosseinzadeh H, Khaleghpanah P. Study of antidepressant effect of aqueous and ethanol extract of Crocus sativus in mice. Iranian J Basic Med Sci 2001:18690.

3. Moshiri E, Basti AA, Noorbala AA, et al. Crocus sativus L. (petal) in the treatment of mild-to-moderate depression: a doubleblind, randomized and placebocontrolled trial. Phytomedicine 2006;9-10:607-11.

4. Akhondzadeh Basti A, Moshiri E, Noorbala AA, et al. Comparison of petal of Crocus sativus L. and fluoxetine in the treatment of depressed outpatients: a pilot doubleblind randomized trial. Prog Neuropsychopharmacol Biol Psychiatry 2007;31:439-42.

5. Akhondzadeh Basti A, Ghoreishi SA, Noorbala AA, et al. Petal and stigma of
Crocus sativus L. in the treatment of depression: a pilot double-blind randomized trial. J Med Plants 2008;7:29-36.

6. Hosseinzadeh H, Younesi H. Petal and stigma extracts of Crocus sativus L. have antinoceceptive and anti-inflammatory effects in mice. BMC Pharmacol 2002;2:7.

7. Hosseinzadeh H, Ghenaati J. Evaluation of the antitussive effect of stigma and petals of saffron (Crocus sativus) and its components, safranal and crocin in guinea pigs. Fitoterapia 2006;77:446-8.

8. Hosseinzadeh H, Motamedshariaty V, Hadizadeh F. Antidepressant effect of kaempferol, a constituent of saffron (Crocus sativus) petal, in mice and rats. Pharmacology 2007:367-70.

9. Fatehi M, Rashidabady T, FatehiHassanabad Z. Effects of Crocus sativus petals extract on rat blood pressure and on response induced by electrical field stimulation in the rat isolated vas deferens and guinea-pig ileum. J Ethnopharmacology 2003;84:199-203.

10. Salomi MJ, Nair SC, Panikkar KR. Inhibitory effects of igella sativa and Crocus sativus on chemical carcinogenesis in mice. Nutr Cancer 1991;16:67-72.

11. Nair SC, Salomi MJ, Varghese CD, et al. Effect of saffron on thymocyte proliferation, intracellular glutathione levels and its antitumor activity. Bio Factors 1992;4: 51-4.

12. Isao K, Ikuyo KH. Flavonols from saffron flowers, tyrosinase activity and inhibition mechanism. J Agric Food Chem 1999;47: 4121-5.

13. Garrido-Pol JL, Diez de Bethencourt C, Revilla E. Flavonoid's composition of hydrolyzed petal extracts of Crocus sativus. Anal Brometal 1987;39:69-77.

14. Parr AJ, Bolwell GP. Phenols in the plant and in man. J Sci Food Agric 2000;80:9851012.

15. Parr, A J,Bolwell GP. Phenols in the plant and in man. The potential for possible nutritional enhancement of the diet by modifying the phenols content or profile. J
Sci Food Agric 2000;80:985-1012.

16. Esmaeili N, Ebrahimzadeh H, Abdi K, et al. Determination of some phenolic compounds in Crocus sativus L. corms and its antioxidant activities study. Phcog Mag 2011;7:74-80.

17. Heim T, Bobilya A. Flavonoids antioxidants: chemistry, metabolism and structure activity relationships. J Nutr Biochem 2002;13:572-84.

18. Ohkawa H, Ohishi N, Yagi K. Assay for lipid peroxides in animal tissues by thiobarbituric acid reaction. Anal Biochem 1979;95:351-8.

19. Sedlak J, Lindsay RH. Estimation of total, protein bound and non protein bound sulfhydryl groups in tissues with Ellman's reagent. Anal Biochem 1968;25:192-205.

20. Benzie IF, Strain JJ. Ferric reducing/ antioxidant power assay: direct measure of total antioxidant activity of biological fluids and modified version for simultaneous measurement of total antioxidant power and ascorbic acid concentration. Method Enzymol 1999;299:15-27.

21. Mansour MM, Farid Z, Bassily S, et al. Serum enzyme tests in hepatosplenicschistosomiasis. Trans Royal Soci Tropic Med Hyg 1982;76:109-11.

22. Norusis MY. SPSS for windows base system user's guide release 6.0., 1st edn. Michigan: SPSS Inc; 1993. pp 281-290.

23. Grune T, Berger MM. Markers of oxidative stress in ICU clinical settings: present and future. Curr Opin Clin Nutr Metab Care 2007;10:712-72.

24. Hossein Goli SA, Mokhtari F, Rahimmalek M. Phenolic compounds and antioxidant activity from saffron (crocus sativus L.) petal. J Agri Sci 2012;4:175.

25. Hadizadeh F, Khalilia, N, Hosseinzadeh $\mathrm{H}$, et al. Kaempferol from saffron petals. Iran J Pharmaceuti Res 2003;2:251-2.

26. Chia-Ying L, E-Jian L, Tian-Shung W. Antityrosinase principles and constituents of the petals of Crocus sativus. J Natur Products 2004;67:437-40. 